\title{
A Uniqueness Theorem for Systems of Interacting Polymers
}

\author{
Volker Warstat \\ Sektion Mathematik, Martin-Luther-Universität Halle-Wittenberg, DDR-4020 Halle, \\ German Democratic Republic
}

\begin{abstract}
In this paper we study analyticity properties of the pressure of general polymer systems by application of Ruelle's theorem on the zeros of Asano contracted polynomials to the partition function. Then we prove a uniqueness theorem for the equilibrium state of the investigated class of classical particle systems for a variety of finite range interactions.
\end{abstract}

\section{Introduction}

Lattice models are still an effective tool to solve phase transition problems in classical statistical mechanics. In the centre of interest we find models without restrictions. In these models the particles of the system can occupy one point of the lattice. But there were moreover a lot of attempts to develop models for extended particles that cover more than one point without penetrating each other. Especially hard rod models have been widely used to explain the existence of liquid crystals. Thus the theory of monomer-dimer systems is extensively elaborated. We refer the reader to [6] for a brief historical sketch that shows, how monomer-dimer systems are related to physics and chemistry.

In $[5,6]$ the absence of a phase transition is proved for translationally invariant systems of noninteracting monomers and dimers. In [1, 7] Abraham and Heilmann respectively Heilmann and Lieb proved with the help of reflection positivity arguments the existence of a phase transition for some systems of interacting monomers and dimers. We will mention here also the papers $[8,9,12]$, that contain results for monomer-dimer systems, but their assumptions on the interactions are very artificial.

Gruber and Kunz incorporated more complicated with respect to their geometry particles into their model in [5]. They showed the absence of a phase transition for noninteracting polymers and thus generalized the results of [6]. We are going to introduce and to investigate a very general model for interacting polymers on the lattice $\mathbb{Z}^{d}$. Under the assumption of $\mathbb{Z}^{d}$-invariance for such polymer systems a variational principle for the pressure $P(J)$ of an interaction $J$ is 
proved in [14] in the form

$$
P(J)=\sup _{\mu \in \mathbb{E}}\left|\mathscr{G}_{0}\right|^{-1}(h(\mu)-\mu(J)) .
$$

In this formula $h(\mu)$ denotes the entropy of the $\mathbb{Z}^{d}$-invariant state $\mu$ and $\mu(J)$ denotes the specific energy of the system. $\left|\mathscr{G}_{0}\right|$ is the number of different shapes, that polymers are allowed to have. States $\mu$, for which the value $\left|\mathscr{G}_{0}\right|^{-1}(h(\mu)-\mu(J))$ equals the pressure $P(J)$, are called equilibrium states for $J$. It is well known that the problem of the uniqueness of the equilibrium state, i.e. the question for the absence of a phase transition, is closely related to the question for analyticity properties of the pressure. A powerful tool to answer the last question is the AsanoRuelle contraction method, that originates in the famous Lee-Yang theorems. We use this method to find regions for the values $J\left(\left\{G_{1}, \ldots, G_{n}\right\}\right)$, the interaction of the set $\left\{G_{1}, \ldots, G_{n}\right\}$ of polymers, where the partition functions do not vanish, and hence the pressure is analytic. The main result (see Theorem 4.1 for the exact formulation) is the following: Under the assumption $J(\{G\}) \neq 0$ for all polymers $G$, i.e. under the assumption that each polymer will give a contribution to the hamiltonian, for small enough values of $\left|e^{-2 J\left(\left\{G_{1}, \ldots, G_{n}\right\}\right)}\right|$ for each polymer set $\left\{G_{1}, \ldots, G_{n}\right\}$ with $J\left(\left\{G_{1}, \ldots, G_{n}\right\}\right) \neq 0$, the pressure is analytic, and hence the equilibrium state for $J$ is unique.

In the case of positive interactions $J$ of the form $J\left(\left\{G_{1}, \ldots, G_{n}\right\}\right)$ $=\frac{1}{k T} J^{\prime}\left(\left\{G_{1}, \ldots, G_{n}\right\}\right)$, where $k$ is the Boltzmann constant and $T$ is the temperature, the theorem states the uniqueness of the equilibrium state in the low temperature region. This agrees with some results in $[5,6]$. Moreover it extends them to a much wider class of interactions by allowing the case $J\left(\left\{G_{1}, \ldots, G_{n}\right\}\right) \neq 0$ for $n>1$, i.e. by allowing attractive or repulsive interactions between the particles.

In Sect. 2 we recall the construction of the configuration space, introduce interactions and formulate exactly the variational principle. In Sect. 3 the algebraic tools for the proof of the main theorem are prepared. In Sect. 4 the uniqueness theorem will be stated and proved. Then we apply it to a system of interacting dimers.

\section{The Model}

Ruelle developed a rather general method for the construction of configuration spaces of classical lattice systems in [11]. In specifying his setting we are able to give a general approach to polymer systems, that contains monomer-dimer systems as well as more complicated systems on the lattice $\mathbb{Z}^{d}$, the set of all $d$-tuples of integers. As all systems will be assumed to be invariant under shift transformations, we define the group $\Theta .=\left\{\Theta_{s} \mid s \in \mathbb{Z}^{d}\right\}$ of shift transformations on $\mathbb{Z}^{d}$ by:

$$
\begin{aligned}
\Theta_{s} t & =t-s \quad\left(s, t \in \mathbb{Z}^{d}\right) \\
\Theta_{s} V & =\left\{t \in \mathbb{Z}^{d} \mid t+s \in V\right\} \quad\left(s \in \mathbb{Z}^{d}, V \subset \mathbb{Z}^{d}\right) \\
\Theta_{s} \mathscr{V} & =\left\{W \subset \mathbb{Z}^{d} \mid \exists V \in \mathscr{V} \quad \text { such that } W=\Theta_{s} V\right\}, \quad\left(s \in \mathbb{Z}^{d}, \mathscr{V} \subset \mathfrak{P}\left(\mathbb{Z}^{d}\right)\right),
\end{aligned}
$$

where $\mathfrak{P}\left(\mathbb{Z}^{d}\right)$ denotes the set of all subsets of $\mathbb{Z}^{d}$. 
We describe now the construction of the configuration space for polymer systems. Each polymer will have the shape of a finite subset of the lattice. Thus we choose for each polymer system a finite set $\mathscr{G}_{0}$ of possible polymer shapes in the following way:

(1) $\mathscr{G}_{0}=\left\{G_{0}^{\prime}, G_{0}^{\prime \prime}, \ldots, G_{0}^{(n)}\right\}$ consists of finite subsets of $\mathbb{Z}^{d}$.

(2) The origin of $\mathbb{Z}^{d}$ is the smallest element of $G_{0}$ in the lexicographic order for each $G_{0} \in \mathscr{G}_{0}$.

From the set $\mathscr{G}_{0}$ the set $\mathscr{G}$ is uniquely generated by shifting $\mathscr{G}_{0}$ over the lattice $\mathbb{Z}^{d}$. In the following $\mathfrak{P}(\mathscr{G})$ denotes the set of all subsets of $\mathscr{G}$, while $\mathfrak{P}_{f}(\mathscr{G})$ comprises all finite elements of $\mathfrak{P}(\mathscr{G})$. For all polymer arrangements on the lattice it is assumed that the particles do not penetrate each other. This leads to the following definition.

2.1. Definition. Given a set $\mathscr{G}_{0}$ with the properties (1) and (2), the polymer configuration space $\Omega$ for $\mathscr{G}_{0}$ is defined by

$$
\Omega=\{\mathscr{W} \in \mathfrak{P}(\mathscr{G}) \mid G, K \in \mathscr{W} \Rightarrow G \cap K=\emptyset\} .
$$

For $\mathscr{V} \in \mathfrak{P}_{f}(\mathscr{G})$ denotes

$$
X_{\mathscr{V}} \Omega=\{\mathscr{Y} \subset \mathscr{V} \mid \exists \mathscr{W} \in \Omega \text { such that } \mathscr{Y} \subset \mathscr{W}\} .
$$

The dependence of $\Omega$ on the choise of $\mathscr{G}_{0}$ will be omitted because there cannot arise any confusion.

The connection with the ideas of Ruelle in [11] will become obvious, if one identifies $\Omega$ with

$$
\begin{array}{r}
\Omega^{*}=\left\{\omega \in \underset{G \in \mathscr{G}}{X}\{0,1\} \mid \text { for each } s \in \mathbb{Z}^{d}\right. \text { there exists at most } \\
\text { one } \left.G \in \mathscr{G} \text { with } s \in G \text { and } X_{\{G\}} \omega \neq 0\right\} .
\end{array}
$$

In this formula $X_{\{G\}}$ denotes the projection operator onto the set $\{G\}$. In this context it is easy to see that $\Omega^{*}$, and thus $\Omega$ will become a compact space, if one starts with the discrete topology on $\{0,1\}$ and uses as the topology on $\Omega^{*}$ the trace topology of the Tychonow-topology on $\underset{G \in \mathscr{G}}{X}\{0,1\}$.

The above construction includes the case of classical lattice gases. One has to choose $\mathscr{G}_{0}=\{\{0\}\}$, where 0 is the origin of $\mathbb{Z}^{d}$. In the following this case will be excluded.

States are regular Borel probability measures on $\Omega$. The set of $\Theta$-invariant states is denoted by $\mathbb{E}$.

2.2. Definition. An interaction is a complex valued function $J$ on the nonempty finite subsets of $\mathscr{G}$. The elements in the support of $J$ are called bonds, i.e. the set of bonds for the interaction $J$ is

$$
\mathfrak{B}=\left\{\mathscr{B} \in \mathfrak{B}_{f}(\mathscr{G}) \mid J(\mathscr{B}) \neq 0\right\} .
$$

If $\mathscr{V} \in \mathfrak{P}_{f}(\mathscr{G})$, then $\mathfrak{B}_{\mathscr{V}}=\{\mathscr{B} \in \mathfrak{B} \mid \mathscr{B} \subset \mathscr{V}\}$.

In what follows we consider only $\Theta$-invariant interactions of finite range. This means that the set of bonds is generated by the group of shifts from a finite set $\mathfrak{B}_{0}$ with the property $\mathscr{B}_{0} \cap \mathscr{G}_{0} \neq \emptyset$ for each $\mathscr{B}_{0} \in \mathfrak{B}_{0}$. 
2.3. Definition. The partition function for the subsystem in $\mathscr{V} \in \mathfrak{P}_{f}(\mathscr{G}), \mathscr{V} \neq \emptyset$, is given by

$$
Z_{\mathscr{V}}(J)=\sum_{\mathscr{W} \in X_{\mathscr{V}} \Omega} \exp \left(\sum_{\mathscr{B} \in \mathfrak{B}_{\mathscr{V}}} J(\mathscr{B})(-1)^{|\mathscr{W} \cap \mathscr{B}|}\right) .
$$

Here $|\mathscr{W} \cap \mathscr{B}|$ denotes the cardinality of the set $\mathscr{W} \cap \mathscr{B}$.

At this point it might be that confusion arises, because this is in general the method to describe spin interactions rather than particle interactions. But because of the $\Theta$-invariance and the finite range of the interactions the above description is in fact no restriction of generality.

Let for $a \in \mathbb{Z}^{+d}=\left\{\left(s_{1}, \ldots, s_{d}\right) \in \mathbb{Z}^{d} \mid s_{i} \geqq 0, i=1, \ldots, d\right\}$ denote

$$
V(a)=\left\{\left(s_{1}, \ldots, s_{d}\right) \in \mathbb{Z}^{d} \mid-a_{i} \leqq s_{i} \leqq a_{i}, i=1, \ldots, d\right\}
$$

and

$$
\mathscr{V}(a)=\{G \in \mathscr{G} \mid G \cap V(a) \neq \emptyset\} .
$$

For $V \subset \mathbb{Z}^{d}$ respectively $\mathscr{V} \subset \mathscr{G},|V|$ respectively $|\mathscr{V}|$ denotes the cardinality of the corresponding set.

In $[14]$ the existence of the thermodynamic limit

$$
\lim _{a \rightarrow \infty}|\mathscr{V}(a)|^{-1} \log Z_{\mathscr{V}(a)}(J)=P(J)
$$

is proved for all $\Theta$-invariant finite range interactions $J$. The limit $P(J)$ is called the pressure of the system. It is known (see [14]) that the map $P: J \rightarrow P(J)$ is monotone increasing, continuous and convex.

For a $\Theta$-invariant state $\mu$ and an interaction $J$ the specific energy is denoted by $\mu(J)$, while the entropy is denoted by $h(\mu)$. These two quantities are defined by the following limits that, indeed, exist (see [14]):

$$
\begin{gathered}
\mu(J)=\lim _{a \rightarrow \infty}-|V(a)|^{-1} \int\left(\sum_{\mathscr{B} \in \mathfrak{B}_{\mathscr{V}(a)}} J(\mathscr{B})(-1)^{|\mathscr{W} \cap \mathscr{B}|}\right) d \mu(\mathscr{W}), \\
h(\mu)=\lim _{a \rightarrow \infty}-|V(a)|^{-1} \sum_{\mathscr{W} \in X_{\mathscr{V}(a) \Omega}}\left(X_{\mathscr{V}(a)} \mu\right)(\mathscr{W}) \log \left(X_{\mathscr{V}(a)} \mu\right)(\mathscr{W}) .
\end{gathered}
$$

We now quote from [14] the following theorem:

2.4. Theorem. For each shift invariant finite range interaction $J$, we have

$$
P(J)=\sup _{\mu \in \mathfrak{E}}\left|\mathscr{G}_{0}\right|^{-1}(h(\mu)-\mu(J)) .
$$

The elements of

$$
\mathfrak{E}_{J}=\left\{\left.\mu \in \mathbb{E}|P(J)=| \mathscr{G}_{0}\right|^{-1}(h(\mu)-\mu(J))\right.
$$

are the equilibrium states of $J$. The set $\mathfrak{E}_{J}$ is nonempty, convex, compact and equals the set

$$
\mathfrak{E}_{J}^{\prime}=\left\{\left.\mu \in \mathbb{E}|P(J+\bar{J}) \geqq P(J)-| \mathscr{G}_{0}\right|^{-1} \mu(\bar{J}) \text { for all } \bar{J}\right\} .
$$

The problem of phase transitions in statistical mechanics for such polymer systems reduces now to the question: What are conditions on the interaction $J$ that 
allow a rigorous proof of $\left|\mathfrak{E}_{J}\right|>1$ or $\left|\mathfrak{E}_{J}\right|=1$ ? In the following we prepare a result concerning the above case $\left|\mathfrak{E}_{J}\right|=1$. For this we introduce the correlation functions of a state.

2.5. Definition. If $\mu$ is a state, then the values

$$
\mu\left(\sigma_{\mathscr{W}}\right)=\int \sigma_{\mathscr{W}}(\mathscr{Y}) d \mu(\mathscr{Y}) \quad\left(\mathscr{W} \in \mathfrak{P}_{f}(\mathscr{G})\right)
$$

with $\sigma_{\mathscr{W}}(\mathscr{Y})=(-1)^{|\mathscr{W} \cap \mathscr{Y}|}$ are called the correlation functions of the state $\mu$.

It follows easily (see also [3]) that each state $\mu$ is uniquely determined by the set of correlation functions $\mu\left(\sigma_{w}\right)$ for finite $\mathscr{W} \in \Omega$. The next theorem allows us to calculate the correlation functions for the equilibrium states of a given interaction $J$ and to answer the question whether $\left|\mathfrak{E}_{J}\right|=1$.

2.6. Theorem. Let $J$ be an interaction and define for each finite $\mathscr{W} \in \Omega$ the interaction $J_{\mathscr{W}}$ by

$$
J_{\mathscr{W}}(\mathscr{V})= \begin{cases}\left|\mathscr{G}_{0}\right| & \text { if } \mathscr{V}=\Theta_{s} \mathscr{W} \text { for some } s \in \mathbb{Z}^{d} \\ 0 & \text { otherwise. }\end{cases}
$$

Then, if the map $t \rightarrow P\left(J+t J_{\mathscr{W}}\right)$ is differentiable at the point $t=0$, the value $\mu\left(\sigma_{\mathscr{W}}\right)$ is the same for all equilibrium states $\mu$ of $J$ and

$$
\mu\left(\sigma_{\mathscr{W}}\right)=\left.\frac{d}{d t} P\left(J+t J_{\mathscr{W}}\right)\right|_{t=0} .
$$

Proof. From the convexity of the pressure (see 2.3) and formula (6) follows that it is sufficient to show that each equilibrium state $\mu$ of $J$ fulfills the following equation:

$$
-\left|\mathscr{G}_{0}\right|^{-1} \mu\left(J_{\mathscr{W}}\right)=\mu\left(\sigma_{\mathscr{W}}\right) \quad(\mathscr{W} \in \Omega, \text { finite }) .
$$

From the definition of $J_{\mathscr{W}}$ we have that $\mathfrak{B}_{0}$ is a one point set. The element of $\mathfrak{B}_{0}$ is denoted by $\mathscr{W}_{0}$. Now we use formula (3) and the $\Theta$.-invariance of $\mu$ and calculate:

$$
\begin{aligned}
-\left|\mathscr{G}_{0}\right|^{-1} \mu\left(J_{\mathscr{W}}\right) & =-\left(\lim _{a \rightarrow \infty}-|V(a)|^{-1}\left|\mathscr{G}_{0}\right|^{-1} \int \sum_{\mathscr{B} \in \mathfrak{B}_{\mathscr{V}(a)}} J_{\mathscr{W}}(\mathscr{B}) \sigma_{\mathscr{B}}(\mathscr{Y}) d \mu(\mathscr{Y})\right) \\
& =\lim _{a \rightarrow \infty}|V(a)|^{-1} \int \sum_{\substack{s \in \mathbb{Z}^{d} \\
\Theta_{s} \mathscr{W}_{0} \subset \mathscr{V}(a)}} \sigma_{\Theta_{s} \mathscr{W}_{0}}(\mathscr{Y}) d \mu(\mathscr{Y}) \\
& =\mu\left(\sigma_{\mathscr{W}_{0}}\right) \lim _{a \rightarrow \infty} \frac{\left|\left\{s \in \mathbb{Z}^{d} \mid \Theta_{s} \mathscr{W}_{0} \subset \mathscr{V}(a)\right\}\right|}{|V(a)|} \\
& =\mu\left(\sigma_{\mathscr{W}}\right) .
\end{aligned}
$$

The above theorem is the motivation to look for differentiability properties of the pressure, i.e. of

$$
P(J)=\lim _{a \rightarrow \infty}|\mathscr{V}(a)|^{-1} \log Z_{\mathscr{V}(a)}(J)
$$

in dependence of the values $J\left(\mathscr{B}_{0}\right)$ with $\mathscr{B}_{0} \in \mathfrak{B}_{0}$. That's why one is at first looking for regions, where the partition functions do not vanish. This can be done with the help of the well known Asano-Ruelle contraction method for polynomials (see $[2,10])$, because of the possibility to write the partition functions $Z_{\mathscr{V}(a)}(J)$ as 
polynomials in the variables $z_{\mathscr{B}}=e^{-2 J(\mathscr{B})}$ with $\mathscr{B} \in \mathfrak{B}_{\mathscr{V}(a)}$. In the next section we will make this precise.

\section{Asano Contraction and Zeros of the Partition Functions}

In this section we introduce and use some algebraic operations, that allow us to write the partition functions $Z_{\mathscr{V}(a)}(J)$ as polynomials in the variables $z_{\mathscr{B}}=e^{-2 J(\mathscr{B})}$ with $\mathscr{B} \in \mathfrak{B}_{\mathscr{V}(a)}$. This is necessary for the application of the Asano-Ruelle contraction method for polynomials. We use this method to find regions for the values of $z_{\mathscr{B}}$, where the partition functions $Z_{\mathscr{V}(a)}(J)$ do not vanish.

Some of the algebraic operations will be used later in different contexts. That's why they will be introduced in a general setting. Let $H$ be a countable set, for example $\mathfrak{P}_{f}(\mathscr{G})$. Then the set $\mathfrak{P}(H)$ of all subsets of $H$ is an abelian group with the operation of symmetric difference. The symmetric difference of two subsets of $H$ is defined by

$$
M \cdot N=(M \cup N) \backslash(M \cap N) \quad(M, N \subset H) .
$$

If $\mathfrak{P}_{f}(H)$ is the set of all finite subsets of $H$, then one can define for each $M \in \mathfrak{P}_{f}(H)$ the map

$$
\sigma_{M}: \mathfrak{P}(H) \rightarrow\{-1,+1\} \quad \text { with } \quad \sigma_{M}(N)=(-1)^{|M \cap N|},
$$

where $|M \cap N|$ denotes the cardinality of the set $M \cap N$. Let $\mathfrak{N}$ be a subset of $\mathfrak{P}_{f}(H)$, then $\overline{\mathfrak{N}}$ denotes the subgroup of $\mathfrak{P}_{f}(H)$ that is generated by $\mathfrak{N}$. The set $\mathfrak{N}^{\perp}$ is defined by

$$
\mathfrak{N}^{\perp}=\left\{M \in \mathfrak{P}(H) \mid \sigma_{N}(M)=1 \text { for all } N \in \mathfrak{N}\right\} .
$$

For the sake of completeness we quote from [10]:

3.1. Definition. Let $H$ be some finite set and let $\left\{H_{i}\right\}$ be a finite covering of $H$. With each $h \in H$ we associate a complex variable $z_{h}$. The polynomial

$$
\mathbb{P}\left(z_{H}\right)=\sum_{M \subset H} c_{M} \prod_{h \in M} z_{h}
$$

is the Asano contraction of the family of polynomials

$$
\left\{\mathbb{P}_{i}\left(z_{H_{i}}\right)=\sum_{M_{i} \subset H_{i}} c_{i, M_{i}} \prod_{h \in M_{i}} z_{h}\right\},
$$

if for all $M \subset H$ holds $c_{M}=\prod_{i} c_{i, M \cap H_{i}}$.

A variable $z_{h}$ is said to undergo contraction, if it belongs to more than one $H_{i}$.

3.2. Theorem [10]. Let $H,\left\{H_{i}\right\}$ and $z_{h}$ be as in 3.1. Let further $R_{i, h}$ with $h \in H_{i}$ be closed subsets of the complex plane that do not contain the origin, if $h$ undergoes contraction. Then, if $\mathbb{P}\left(z_{H}\right)$ is the Asano contraction of the polynomials $\left\{\mathbb{P}_{i}\left(z_{H_{i}}\right)\right\}$, and if $\mathbb{P}_{i}\left(z_{H_{1}}\right) \neq 0$ holds when $z_{h} \notin R_{i, h}$ for all $h \in H_{i}, \mathbb{P}\left(z_{H}\right) \neq 0$ holds when $z_{h} \notin \prod_{i}^{*}\left(-R_{i, h}\right)$ for all $h \in H$.

For two subsets $R^{\prime}, R^{\prime \prime} \subset \mathbb{C}$ the product $*$ is defined as $R^{\prime} * R^{\prime \prime}$ $=\left\{r^{\prime} \cdot r^{\prime \prime} \in \mathbb{C} \mid r^{\prime} \in R^{\prime}, r^{\prime \prime} \in R^{\prime \prime}\right\}$. For $R \subset \mathbb{C}$ the set $-R$ is defined by $\{-r \in \mathbb{C} \mid r \in R\}$. 
The following theorem allows us to decide in special cases, that are closely related to the case of partition functions, whether a given polynomial is the Asano contraction of a given family of polynomials.

3.3. Theorem [4]. Let $H$ be an arbitrary finite set and $\left\{H_{i}\right\}$ be a finite covering of $H$. If for $\mathfrak{M} \subset \mathfrak{P}(H)$ and for the system $\left\{\mathfrak{M}_{i}=\left\{M \cap H_{i} \mid M \in \mathfrak{M}\right\}\right\}$ the conditions

(1) For any $M \in \overline{\mathfrak{M}}$ the condition $M \cap H_{i} \in \mathfrak{M}_{i}$ for all $i$ imply $M \in \mathfrak{M}$;

(2) The subgroup of $\mathfrak{P}(H)$ that is generated by the system $\left\{\mathfrak{M}_{i}^{\perp}=\mathfrak{M}^{\perp} \cap \mathfrak{P}\left(H_{i}\right)\right\}$ coincides with $\mathfrak{M}^{\perp}$;

hold, then the polynomial

$$
\mathbb{P}\left(z_{H}\right)=\sum_{M \in \mathfrak{M}} \prod_{h \in M} z_{h}
$$

is the Asano contraction of the polynomials

$$
\left\{\mathbb{P}_{i}\left(z_{H_{l}}\right)=\sum_{M \in \mathfrak{M}_{i}} \prod_{h \in M} z_{h}\right\}
$$

We want to apply this theory to polymer systems. For this we define for $\mathscr{V} \in \mathfrak{P}_{f}(\mathscr{G})$ the group homomorphisms $\pi_{\mathscr{V}}$ and $\gamma_{\mathscr{V}}$ by

$$
\begin{aligned}
& \pi_{\mathscr{V}}: \mathfrak{P}\left(\mathfrak{B}_{\mathscr{V}}\right) \rightarrow \mathfrak{P}(\mathscr{V}) \quad \text { with } \quad \pi_{\mathscr{V}}(\beta)=\prod_{\mathscr{B} \in \beta} \mathscr{B}, \\
& \gamma_{\mathscr{V}}: \mathfrak{P}(\mathscr{V}) \rightarrow \mathfrak{P}\left(\mathfrak{B}_{\mathscr{V}}\right) \quad \text { with } \quad \gamma_{\mathscr{V}}(\mathscr{W})=\left\{\mathscr{B} \in \mathfrak{B}_{\mathscr{V}} \mid \sigma_{\mathscr{B}}(\mathscr{W})=-1\right\} .
\end{aligned}
$$

We denote $\mathfrak{R}_{\mathscr{V}}=\operatorname{ker} \pi_{\mathscr{V}}$.

With these notations we can calculate (see formula (1)):

$$
\begin{aligned}
Z_{\mathscr{V}}(J) & =\sum_{\mathscr{W} \in X_{\mathscr{V}} \Omega} \exp \left(\sum_{\mathscr{B} \in \mathfrak{B}_{\mathscr{V}}} J(\mathscr{B})(-1)^{|\mathscr{W} \cap \mathscr{B}|}\right) \\
& =\sum_{\mathscr{W} \in X_{\mathscr{V}} \Omega} \prod_{\mathscr{B} \in \mathfrak{B}_{\mathscr{V}}} \exp \left(J(\mathscr{B}) \sigma_{\mathscr{B}}(\mathscr{W})\right) \\
& =\sum_{\mathscr{W} \in X_{\mathscr{V}} \Omega} \prod_{\mathscr{B} \in \mathfrak{B}_{\mathscr{V}}} \exp \left(-J(\mathscr{B})\left[1-\sigma_{\mathscr{B}}(\mathscr{W})\right]\right) \prod_{\mathscr{B} \in \mathfrak{B}_{\mathscr{V}}} \exp (J(\mathscr{B})) \\
& =\prod_{\mathscr{B} \in \mathfrak{B}_{\mathscr{V}}} \exp (J(\mathscr{B}))\left|\operatorname{ker} \gamma_{\mathscr{V}}\right| \sum_{\beta \in \mathcal{Y}_{\mathscr{V}}\left(X_{\mathscr{V}} \Omega\right)} \prod_{\mathscr{B} \in \beta} \exp (-2 J(\mathscr{B})) .
\end{aligned}
$$

The factor $\prod_{\mathscr{B} \in \mathfrak{B}_{\mathscr{V}}} \exp (J(\mathscr{B}))\left|\operatorname{ker} \gamma_{\mathscr{V}}\right|$ does not influence the behaviour of the zeros of the partition function. So it will be omitted, and we will continue with the examination of

$$
Z_{\mathscr{V}}^{\mathrm{red}}(J)=\sum_{\beta \in \gamma_{\mathscr{V}}\left(X_{\mathscr{V}} \Omega\right)} \prod_{\mathscr{B} \in \beta} \exp (-2 J(\mathscr{B}))
$$

This is obviously a polynomial in the variables $z_{\mathscr{B}}=e^{-2 J(\mathscr{B})}$, that looks like the polynomials in the above theorem. The set $\mathfrak{M}$ in 3.3 is now $\gamma_{\mathscr{v}}\left(X_{\mathscr{v}} \Omega\right)$. For this set we can prove:

3.4. Proposition. For each polymer system holds

$$
\left[\gamma_{\mathscr{V}}\left(X_{\mathscr{V}} \Omega\right)\right]^{\perp}=\mathfrak{R}_{\mathscr{V}}=\operatorname{ker} \pi_{\mathscr{V}}, \quad\left(\mathscr{V} \in \mathfrak{P}_{f}(\mathscr{G})\right) .
$$


Proof. By the definition of the operation ${ }^{\perp}$ [see (8)], we have for $\mathscr{V} \in \mathfrak{P}_{f}(\mathscr{G})$,

$$
\begin{aligned}
{\left[\gamma_{\mathscr{V}}\left(X_{\mathscr{V}} \Omega\right)\right]^{\perp}=} & \left\{\left(\mathscr{B}_{1}, \ldots, \mathscr{B}_{n}\right) \mid \mathscr{B}_{i} \in \mathfrak{B}_{\mathscr{V}} \quad \text { with } \quad \sigma_{\left(\mathscr{B}_{1}, \ldots, \mathscr{B}_{n}\right)}\left(\gamma_{\mathscr{V}}(\mathscr{W})\right)=1\right. \\
& \text { for all } \left.\mathscr{W} \in X_{\mathscr{V}} \Omega\right\} \\
= & \left\{\left(\mathscr{B}_{1}, \ldots, \mathscr{B}_{n}\right) \mid \mathscr{B}_{i} \in \mathfrak{B}_{\mathscr{V}} \quad \text { with } \quad \sigma_{\substack{i=1 \\
i=1}}^{n}(\mathscr{W})=1\right. \\
& \text { for all } \left.\mathscr{W} \in X_{\mathscr{V}} \Omega\right\} .
\end{aligned}
$$

For each set $\left(\mathscr{B}_{1}, \ldots, \mathscr{B}_{n}\right) \in \mathfrak{R}_{\mathscr{r}}$ holds $\prod_{i=1}^{n} \mathscr{B}_{i}=\emptyset$, and thus $\left(\mathscr{B}_{1}, \ldots, \mathscr{B}_{n}\right)$ $\in\left[\gamma_{\psi}\left(X_{\mathscr{r}} \Omega\right)\right]^{\perp}$.

If on the other hand $\left(\mathscr{B}_{1}, \ldots, \mathscr{B}_{n}\right) \notin \mathfrak{S}_{\mathscr{V}}$, then $\emptyset \neq \prod_{i=1}^{n} \mathscr{B}_{i}=\overline{\mathscr{B}} \in \mathfrak{P}(\mathscr{V})$. Because of $\{G\} \in X_{\mathscr{Y}} \Omega$ for each $G \in \mathscr{V}$, we find for $G \in \overline{\mathscr{B}}$ that $\sigma_{\overline{\mathscr{B}}}(\{G\})=-1$, i.e. $\left(\mathscr{B}_{1}, \ldots, \mathscr{B}_{n}\right)$ $\notin\left[\gamma_{\mathscr{\gamma}}\left(X_{\mathscr{V}} \Omega\right)\right]^{\perp}$.

Later it will be useful to know what happens with $\Re_{\mathscr{V}}=\left[\gamma_{\mathscr{V}}\left(X_{\mathscr{r}} \Omega\right)\right]^{\perp}$, if one enlarges the set $\mathfrak{B}_{\mathscr{V}}$ by elements of $\overline{\mathfrak{B}}_{\mathscr{r}}$, i.e. if one uses more complicated interactions.

3.5. Proposition. Suppose that $\mathscr{B}_{1}, \ldots, \mathscr{B}_{n} \in \overline{\mathfrak{B}}_{\mathscr{r}}$, and put $\mathfrak{B}_{\mathscr{r}}^{\prime}=\mathfrak{B}_{\mathscr{r}} \cup\left\{\mathscr{B}_{1}, \ldots, \mathscr{B}_{n}\right\}, \mathfrak{\Re}_{\mathscr{r}}^{\prime}$ the kernel of $\pi_{\mathscr{V}}^{\prime}: \mathfrak{P}\left(\mathfrak{B}_{\mathscr{V}}^{\prime}\right) \rightarrow \mathfrak{P}(\mathscr{V})$. Let $\alpha_{1}, \ldots, \alpha_{n} \in \mathfrak{P}\left(\mathfrak{B}_{\mathscr{V}}\right)$ be such that $\mathscr{B}_{i}=\prod_{\mathscr{B} \in \alpha_{i}} \mathscr{\mathscr { B }}$, and denote $\alpha_{i}^{\prime}=\alpha_{i} \cup\left\{\mathscr{B}_{i}\right\}$. Then $\mathfrak{\Omega}_{\mathscr{v}}^{\prime}$ is generated by $\left\{\alpha_{1}^{\prime}, \ldots, \alpha_{n}^{\prime}\right\} \cup \boldsymbol{\Omega}_{\mathscr{r}}$.

A similar proposition was proved by Slawny in [13] for spin-1/2-classical lattice systems. The proof of our proposition proceeds in the same way as in Slawny's paper. The same is true for the next proposition.

3.6. Proposition. Let $\mathscr{V} \in \mathfrak{B}_{f}(\mathscr{G})$, and suppose that $\mathscr{B}_{i} \notin \mathfrak{B}_{\mathscr{V}}$ and $\alpha_{i} \in \mathfrak{P}\left(\mathfrak{B}_{\mathscr{Y}}\right)$ are chosen such that $\mathscr{B}_{i}=\prod_{\mathscr{B} \in \alpha_{1}} \mathscr{B}$ and no proper subset of $\alpha_{i}$ yields $\mathscr{B}_{i}$. Let $J$ be the interaction that determines $\mathfrak{B}$ and $J^{\prime}$ be another interaction that coincides on $\mathfrak{P}_{f}(\mathscr{G})$ with $J$ with the exception of the sets $\mathscr{B}_{i}$ and their translates. Then, if $Z_{\mathscr{Y}}^{\mathrm{red}}(J) \neq 0$ when $\left|e^{-2 J(\mathscr{B}}\right|=\left|z_{\mathscr{B}}\right|<r_{\mathscr{B}}, \mathscr{B} \in \bigcup_{i} \alpha_{i}$ and $e^{-2 J(\mathscr{B})}=z_{\mathscr{B}} \notin R_{\mathscr{B}}, \mathscr{B} \in \mathfrak{B}_{\mathscr{V}} \bigcup_{i} \alpha_{i}$, for any $\tau \in[0,1]$ $Z_{\mathscr{V}}^{\text {red }}\left(J^{\prime}\right)$ does not vanish when $\left|e^{-2 J^{\prime}(\mathscr{B})}\right|=\left|z_{\mathscr{B}}\right|<\tau^{n_{\mathscr{B}}} \cdot r_{\mathscr{B}}, \mathscr{B} \in \bigcup \alpha_{i}, n_{\mathscr{B}}=\left|\left\{i \mid \mathscr{B} \in \alpha_{i}\right\}\right|$ and $e^{-2 J^{\prime}(\mathscr{B})}=z_{\mathscr{B}} \notin R_{\mathscr{B}}, \quad \mathscr{B} \in \mathfrak{B}_{\mathscr{V}} \backslash \bigcup_{i} \alpha_{i}, \quad$ and $\quad\left|e^{-2 J^{\prime}\left(\mathscr{O}_{i}\right)}-1\right|=\left|z_{\mathscr{B}_{i}}-1\right|<\left(\frac{1-\tau}{1+\tau}\right)^{\left|\alpha_{i}\right|}$,
$i=1, \ldots, n$.

\section{Main Theorem}

We now state the main theorem. Before proving it, we will illustrate it with the help of an example from the literature. It is the model I in [7], a model for interacting dimers.

4.1. Theorem. For each polymer configuration space $\Omega$ on the lattice $\mathbb{Z}^{d}$ and each shift invariant finite range interaction $J$ with the property

holds:

$$
J(\{G\}) \neq 0 \quad(G \in \mathscr{G})
$$

(1) For each $\mathscr{V} \in \mathfrak{P}_{f}(\mathscr{G})$ and all $\mathscr{B} \in \mathfrak{B}_{\mathscr{V}}$ exist positive real numbers $r_{\mathscr{B}}$ such that the partition function $Z_{\mathscr{V}}^{\text {red }}(J)$ does not vanish for $\left|e^{-2 J(\mathscr{B})}\right|=\left|z_{\mathscr{B}}\right|<r_{\mathscr{B}}$. 
There exist for each $\mathscr{B}_{0} \in \mathfrak{B}_{0}$ positive real numbers $r_{\mathscr{B}_{0}}$ such that in the area $\left|z_{\mathscr{B}_{0}}\right|<r_{\mathscr{B}_{0}}$,

(2) the pressure is an analytic function in the variables $z_{\mathscr{B}_{0}}$,

(3) there exists one and only one equilibrium state $\mu$ for $J$,

(4) the correlation functions of $\mu$ are analytic in the variables $z_{\mathscr{B}_{0}}$.

4.2. Remark. In the case $\mathfrak{B}=\mathscr{G}$, i.e. if there is no interaction between the particles, the above theorem is a special case of the results proved in [5] for polymer systems. So we have to draw attention to examples, where there is an interaction between the particles.

4.3. Example. As mentioned above, systems of hard dimers are of essential interest. We want to examine here a model that was introduced by Heilmann and Lieb in [7]. It is a model for systems of hard dimers on the quadratic lattice $\mathbb{Z}^{2}$ ([7], model I). A vertex of the lattice is either empty or covered by at most one dimer. A dimer covers two vertices that are connected by an edge. For such systems we will examine the following interaction:

$$
\begin{aligned}
J(\{D\})=\beta b>0 & \text { for each dimer } D \in \mathscr{G}, \\
J\left(\left\{D_{1}, D_{2}\right\}\right)=\beta c>0 & \text { for each pair of neighbouring, } \\
& \text { collinear dimers } D_{1}, D_{2} \in \mathscr{G},
\end{aligned}
$$

where $\beta=(k T)^{-1}$ is the inverse temperature.

In this situation it follows from 4.1 that there exists a $\beta_{\mathrm{cr}}>0$, such that for $\beta>\beta_{\mathrm{cr}}$ the equilibrium state is unique, i.e. at low temperature there is no phase transition. We want to compare this result with that in [7]. For this it is necessary to transform the "spin type" interaction $J$ into the "particle type" form used in [7] in such a way that the Gibbs distributions of the finite systems in $\mathscr{V} \in \mathfrak{P}_{f}(\mathscr{G})$ will not change. Equal families of Gibbs distributions describe equal sets of shift invariant Gibbs states, that are by a result of [14] equilibrium states. The desired transformation is the following:

$$
U(\{D\})=2 J(\{D\})+2 \sum_{\substack{D^{\prime} \in \mathscr{G} \\\left\langle D, D^{\prime}\right\rangle}} J\left(\left\{D, D^{\prime}\right\}\right) \text { for each dimer } D \in \mathscr{G} .
$$

The sum runs through all dimers $D^{\prime} \in \mathscr{G}$, that are collinear neighbours of $D$. We use the symbol $\left\langle D, D^{\prime}\right\rangle$ for such pairs.

$$
U\left(\left\{D_{1}, D_{2}\right\}\right)=-4 J\left(\left\{D_{1}, D_{2}\right\}\right) \text { for each pair }\left\langle D_{1}, D_{2}\right\rangle .
$$

The partition function with respect to $U$ for the subsystem in $\mathscr{V} \in \mathfrak{P}_{f}(\mathscr{G})$ is defined by

$$
Z_{\mathscr{V}}(U)=\sum_{\mathscr{W} \in X_{\mathscr{W}} \Omega} \exp -\left(\sum_{D \in \mathscr{W}} U(\{D\})+\sum_{\substack{\left\langle D_{1}, D_{2}\right\rangle \\ D_{1}, D_{2} \in \mathscr{W}}} U\left(\left\{D_{1}, D_{2}\right\}\right)\right) .
$$

By showing that for each $\mathscr{W} \in X_{\mathscr{r}} \Omega$ the sum

$$
\begin{aligned}
& \sum_{D \in \mathscr{V}} J(\{D\})(-1)^{|\mathscr{W} \cap\{D\}|}+\sum_{\substack{\left\langle D_{1}, D_{2}\right\rangle \\
D_{1}, D_{2} \in \mathcal{Y}}} J\left(\left\{D_{1}, D_{2}\right\}\right)(-1)^{\left|\mathscr{W} \cap\left\{D_{1}, D_{2}\right\}\right|} \\
& +\sum_{D \in \mathscr{W}} U(\{D\})+\sum_{\substack{\left\langle D_{1}, D_{2}\right\rangle \\
D_{1}, D_{2} \in \mathscr{W}}} U\left(\left\{D_{1}, D_{2}\right\}\right)
\end{aligned}
$$


is independent of $\mathscr{W} \in X_{\mathscr{V}} \Omega$, we can see that the given transformation is the right one in the sense mentioned above. It was shown in [7] that for low enough temperature there is a phase transition, if

$$
U\left(\left\{D_{1}, D_{2}\right\}\right)=-\beta a<0 \quad \text { for each pair }\left\langle D_{1}, D_{2}\right\rangle
$$

and

$$
U(\{D\})=-\mu \beta<\beta a \text { for each dimer } D \in \mathscr{G} .
$$

In 4.1 is proved the absence of a phase transition for low enough temperature for

$$
U\left(\left\{D_{1}, D_{2}\right\}\right)=-4 \beta c<0 \text { and } U(\{D\})=2(\beta b+2 \beta c)>0 .
$$

In this way we get an almost complete idea of the phase transition behaviour of the two-dimensional dimer model with attraction between neighbouring collinear dimers in the low temperature region.

Similar calculations can be done for the other models examinated in [7] and in [1].

4.4. Proof of Theorem 4.1. From the assumptions follows $\{G\} \in \mathfrak{B}$ for all $G \in \mathscr{G}$, and thus $\overline{\mathfrak{B}}=\mathfrak{P}_{f}(\mathscr{G})$. Let now $\mathscr{V}$ be an arbitrary nonempty finite subset of $\mathscr{G}$. With the help of Theorem 3.3 we will show now that $Z_{\mathscr{V}}^{\text {red }}(J)$ is the Asano contraction of a set of small polynomials. For this we need a covering $\left\{\mathfrak{B}_{\mathscr{V}}^{i}\right\}$ of $\mathfrak{B}_{\mathscr{V}}$. We take

$$
\begin{aligned}
\mathfrak{B}_{\mathscr{V}}^{i}= & \{\{G\},\{K\}\} \quad \text { with } \quad G, K \in \mathscr{V}, G \cap K \neq \emptyset \\
\mathfrak{B}_{\mathscr{V}}^{j}= & \left\{\mathfrak{B},\left\{G_{1}\right\}, \ldots,\left\{G_{n}\right\}\right\} \quad \text { with } \mathscr{B} \in \mathfrak{B}_{\mathscr{V}}, \text { card } \mathscr{B}>1, G_{l} \in \mathscr{V}, \\
& l=1, \ldots, n, \prod_{l=1}^{n}\left\{G_{l}\right\}=\mathscr{B} .
\end{aligned}
$$

Here $i$ runs through the set of all pairs $\{G, K\}$ with the above property, and $j$ runs through the set of all $\mathscr{B} \in \mathfrak{B}_{\mathscr{V}}$ with card $\mathscr{B}>1$. The set $\mathfrak{M}$ in 3.3 is $\gamma_{\mathscr{V}}\left(X_{\mathscr{V}} \Omega\right)$. For the sets $\mathfrak{M}_{i}$ we get immediately: $\mathfrak{M}_{i}=\{\emptyset,\{G\},\{K\}\}$. For the verification of the assumption (1) in 3.3 choose an arbitrary $\beta \in \overline{\mathfrak{M}}=\overline{\gamma_{\mathscr{V}}\left(X_{\mathscr{V}} \Omega\right)}$, i.e. take finitely many sets $\mathscr{W}_{1}, \ldots, \mathscr{W}_{n} \in X_{\mathscr{V}} \Omega$ with $\beta=\mathcal{\gamma}_{\mathscr{V}}\left(\prod_{i=1}^{n} \mathscr{W}_{i}\right)$. It follows $\left\{\{G\} \mid G \in \prod_{i=1}^{n} \mathscr{W}_{i}\right\} \subset \beta$. If now $\beta \cap \mathfrak{B}_{\mathscr{V}}^{i} \in \mathfrak{M}_{i}$ holds for all $i$, then $\prod_{i=1}^{n} \mathscr{W}_{i} \in X_{\mathscr{V}} \Omega$. But this means $\beta \in \mathfrak{M}$. Thus the following implication is true:

$$
\left(\beta \in \overline{\mathfrak{M}} \quad \text { and } \quad \beta \cap \mathfrak{B}_{\mathscr{V}}^{i} \in \mathfrak{M}_{i} \text { for all } i\right) \Rightarrow \beta \in \mathfrak{M} .
$$

Hence, we have

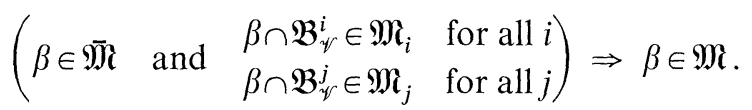

For the verification of the assumption (2) in 3.3 we use Proposition 3.5.

Define a new interaction $\widetilde{J}$ by

$$
\begin{aligned}
\tilde{J}(\{G\}) & =J(\{G\}) & & (G \in \mathscr{G}), \\
\tilde{J}(\mathscr{V}) & =0 & & \left(\mathscr{V} \in \mathfrak{P}_{f}(\mathscr{G}) \backslash \mathscr{G}\right) .
\end{aligned}
$$


Then $\widetilde{\mathfrak{R}}_{\mathscr{r}}=\{\emptyset\}$. Because of $\mathfrak{M}_{i}^{\perp}=\{\emptyset\}$ the kernel $\widetilde{\mathfrak{R}}_{\mathscr{r}}$ is generated by the sets $\mathfrak{M}_{i}^{\perp}$. From 3.4 and 3.5 follows that $\mathfrak{M}^{\perp}=\boldsymbol{\Omega}_{\mathscr{r}}$ is generated by the system $\left\{\mathfrak{M}_{i}^{\perp}\right\}$ and the

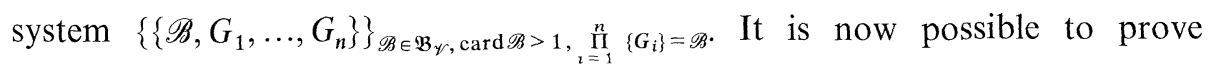
that $\mathfrak{M}_{j}^{\perp}=\left\{\emptyset,\left\{\mathscr{B}, G_{1}, \ldots, G_{n}\right\}\right\}$, i.e. that the condition 3.3 (2) is fulfilled. By definition holds

$$
\mathfrak{M}_{j}^{\perp}=\left\{\beta \in\left\{\mathscr{B}, G_{1}, \ldots, G_{n}\right\} \mid \sigma_{\beta}(\gamma)=1 \quad \text { for all } \quad \gamma \in \mathfrak{M}_{j}\right\} .
$$

Because of $\mathfrak{M}_{j}=\left\{\gamma \in \mathfrak{B}\left(\mathfrak{B}_{\gamma}^{j}\right) \mid \exists \gamma^{\prime} \in \mathfrak{M}\right.$ such that $\left.\gamma=\left\{\mathscr{B}, G_{1}, \ldots, G_{n}\right\} \cap \gamma^{\prime}\right\}$, we conclude $\gamma=\left\{\mathscr{B}, G_{l_{1}}, \ldots, G_{l_{m}}\right\}$ with $m$ odd or $\gamma=\emptyset$. Here $\left\{G_{l_{1}}, \ldots, G_{l_{m}}\right\}$ runs through all $m$-tuples of polymers in $\mathscr{B}$ such that $\left\{G_{l_{1}}, \ldots, G_{l_{m}}\right\} \in X_{\mathscr{V}} \Omega$. Therefore $\gamma=\emptyset$ or card $\gamma$ is even for $\gamma \in \mathfrak{M}_{j}$.

Suppose now $\emptyset \neq \beta \subsetneq\left\{\mathscr{B}, G_{1}, \ldots, G_{n}\right\}$. If $\beta=\left\{\mathscr{B}, G_{l_{1}}, \ldots, G_{l_{m}}\right\}$, then choose $\gamma=\left\{\mathscr{B}, G_{l_{k}}\right\} \in \mathfrak{M}_{j}$ with $k \neq 1, \ldots, m$. It follows $\beta \cap \gamma=\{\mathscr{B}\}$, i.e. $\beta \notin \mathfrak{M}_{j}^{\perp}$. If $\beta=\left\{G_{l_{1}}, \ldots, G_{l_{m}}\right\}$, then choose $\gamma=\left\{\mathscr{B}, G_{l_{m}}\right\} \in \mathfrak{M}_{j}$. It follows $\beta \cap \gamma=\left\{G_{l_{m}}\right\}$, i.e. $\beta \notin \mathfrak{M}_{j}^{\perp}$.

Thus the assumptions of 3.3 are fulfilled, and by this theorem $Z_{\mathscr{V}}^{\text {red }}(J)$ is the Asano contraction of the polynomials

$$
\begin{aligned}
& Z_{i}=1+z_{G}+z_{K} \\
& Z_{j}=\sum_{\beta \in\left\{\mathscr{B}, G_{1}, \ldots, G_{n}\right\} \cap \gamma_{\mathcal{Y}}\left(X_{\mathscr{\gamma}} \Omega\right)} \prod_{\mathscr{B} \in \beta} z_{\tilde{\mathscr{B}}} .
\end{aligned}
$$

Each of these polynomials contains the additive term 1 and hence it does not vanish for small enough values $\left|z_{\mathscr{B}}\right|$. By 3.2 exist positive real numbers $r_{\mathscr{B}}$ for $\mathscr{B} \in \mathfrak{B}_{\mathscr{V}}$ such that $Z_{\mathscr{V}}^{\text {red }}(J)$ does not vanish when $\left|z_{\mathscr{B}}\right|<r_{\mathscr{B}}$. Part (1) of the theorem is proved.

By construction no $\mathscr{B} \in \mathfrak{B}_{\mathscr{V}}$ with card $\mathscr{B}>1$ undergoes contraction. For $\mathscr{B}=\{G\}$ with $G \in \mathscr{G}$ the number of contractions is bounded because of the finite range of $J$. Hence it is possible to choose the numbers $r_{\mathscr{B}}$ independently of $\mathscr{V} \in \mathfrak{B}_{f}(\mathscr{G})$. Furthermore we have by the $\Theta$-invariance of $J$ the possibility to realize that $r_{\mathscr{B}}=r_{\mathscr{B}_{0}}$ for all $\mathscr{B}$ that are translates of $\mathscr{B}_{0} \in \mathfrak{B}_{0}$.

In what follows we look at $Z_{\mathscr{V}}^{\text {red }}(J)$ as a function in the variables $z_{\mathscr{B}_{0}}=e^{-2 J\left(\mathscr{B}_{0}\right)}$ with $\mathscr{B}_{0} \in \mathfrak{B}_{0}$. It is easy to see that

$$
0 \leqq\left|Z_{\mathscr{V}}^{\mathrm{red}}(J)\right|=\left|\sum_{\beta \in \mathcal{V}_{\mathscr{V}}\left(X_{\mathscr{V}} \Omega\right)} \prod_{\mathscr{B} \in \beta} z_{\mathscr{B}}\right| \leqq\left|Z_{\mathscr{V}}^{\mathrm{red}}(\tilde{J} \equiv 0)\right|
$$

for $\left|z_{\mathscr{B}_{0}}\right|<r_{\mathscr{B}_{0}}<1$. In this area each partition function is bounded. It follows the uniform boundedness of $|\mathscr{V}|^{-1} \log Z_{\mathscr{r}}(J)$ in this region. Since the sequence of boundaries $|\mathscr{V}(a)|^{-1} \log Z_{\mathscr{V}(a)}(\tilde{J} \equiv 0)$ converges for $a \rightarrow \infty$, we conclude from Vitali's theorem that the pressure is analytic in the variables $z_{\mathscr{B}_{0}}=e^{-2 J\left(\mathscr{B}_{0}\right)}$ with $\mathscr{B}_{0} \in \mathfrak{B}_{0}$ in the circles $\left|z_{\mathscr{B}_{0}}\right|<r_{\mathscr{B}_{0}}$. Part (2) of the theorem is proved.

The parts (3) and (4) follow directly from 3.3 and 3.5. Each finite $\mathscr{W} \in \Omega$ can be written as $\mathscr{W}=\prod_{G \in \mathscr{W}}\{G\}$ with $G \in \mathscr{G}$. From 3.6 we have that for each $\tau \in[0,1]$ the partition function $Z_{\mathscr{V}}^{\text {red }}\left(J+t J_{\mathscr{W}}\right)$ does not vanish for $\left|z_{G}\right|<\tau r_{G}$ with $\{G\} \in \mathfrak{B}_{0}$ and $\Theta_{s} G \in \mathscr{W}$ for at least one $s \in \mathbb{Z}^{d}$ and $\left|z_{\mathscr{B}}\right|<r_{\mathscr{B}}$ for all the other $\mathscr{B} \in \mathfrak{B}_{0}$ and $\left|z_{\mathscr{W}}-1\right|<\left(\frac{1-\tau}{1+\tau}\right)^{|\mathscr{W}|}$. From this follows the analyticity of $P\left(J+t J_{\mathscr{W}}\right)$ as in the 
proof of (2) in particular for small $t$. Thus by 2.5 and 2.6 the equilibrium state for the interaction $J$ is unique, and the correlation functions are analytic functions in the variables $z_{\mathscr{B}_{0}}$ for $\left|z_{\mathscr{B}_{0}}\right|<r_{\mathscr{B}_{0}}, \mathscr{B}_{0} \in \mathfrak{B}_{0}$.

The theorem is proved.

\section{References}

1. Abraham, D.B., Heilmann, O.J.: Interacting dimers on the simple cubic lattice as a model for liquid crystals. J. Phys. A 13, 1051-1062 (1980)

2. Asano, T.: J. Phys. Soc. Jpn. 29, 350-359 (1970)

3. Gruber, C., Hintermann, A.: Group structure analysis for classical lattice systems with constraints. I. General properties. Physica A 83, 233-256 (1976)

4. Gruber, C., Hintermann, A., Merlini, D.: Group analysis of classical systems. Lecture Notes in Physics, Vol. 60. Berlin, Heidelberg, New York: Springer 1977

5. Gruber, C., Kunz, H.: General properties of polymer systems. Commun. Math. Phys. 22, 133-161 (1971)

6. Heilmann, O.J., Lieb, E.H.: Theory of monomer-dimer systems. Commun. Math. Phys. 25, 190-232 (1972)

7. Heilmann, O.J., Lieb, E.H.: Lattice models for liquid crystals. J. Stat. Phys. 20, 679-693 (1979)

8. Heilmann, O.J., Preaestgaard, E.: Chem. Phys. 24, 119-123 (1977)

9. Lebowitz, J.L., Gallavotti, G.:Phase transitions in binary lattice gases. J. Math. Phys. 12, 1129 (1971)

10. Ruelle, D.: Extension of the Lee-Yang circle theorem. Phys. Rev. Lett. 26, 303-304 (1971)

11. Ruelle, D.: Thermodynamic formalism. Reading, MA: Addison-Wesley 1978

12. Runnels, L.K., Freasier, B.C.: Equilibrium states of a dimer model with angular forces. Commun. Math. Phys. 32, 191-204 (1973)

13. Slawny, J.: Analyticity and uniqueness for spin $\frac{1}{2}$ classical ferromagnetic lattice systems at low temperatures. Commun. Math. Phys. 34, 271-296 (1973)

14. Warstat, V.: In preparation

Communicated by J. Fröhlich

Received October 30, 1984; in revised form June 4, 1985 